\title{
CLE-peptides in Medicago truncatula somatic embryogenesis
}

\author{
Zlydneva N.S.*, Kudriashov A.A., Tvorogova V.E., Lutova L.A. \\ St. Petersburg State University, St. Petersburg, Russia \\ *email: st086125@student.spbu.ru
}

Somatic embryogenesis (SE) is a phenomenon allowing plants to regenerate embryos from somatic cells. For the development of somatic embryos, interactions between various plant growth regulators are extremely important. Small secreted peptide hormones are involved in the intercellular communication and regulate various physiological processes. One of the groups of plant peptide hormones is the CLAVATA3 (CLV3)/EMBRYO SURROUNDING REGION (CLE) family, whose members play an important role in the differentiation of the shoot and root meristems. Several transcription factors from the WOX family were shown to regulate the expression of certain $C L E$ genes, which, in turn, affect the expression of corresponding WOX genes according to the principle of feedback. Most well-known CLE peptides, functioning in shoot and root meristem, repress the expression of WUS and WOX5 genes respectively, but there are also examples of the positive effect of CLE peptides on the expression of WOX genes in lateral meristems and during the development of zygotic embryos. Previously in our research, it was found that the expression level of some CLE genes changes on the background of overexpression of MtWOX9-1 gene, SE stimulator. The aim of our study is to further investigate the connection between CLE genes and MtWOX9-1 and to analyze the role of these CLE peptides in SE. We found that MtCLE18 or MtCLE6 overexpression does not affect SE capacity of calli, whereas MtCLE16 and $M t C L E 08$ overexpression could possibly suppress and stimulate SE respectively. We are going to test the effect of these peptides on MtWOX9-1 using inducible expression. We have also obtained the vector constructions for generating edited plants with MtCLE08 and MtCLE16 loss of function.

Acknowledgments: The research was ssupported by the RFBR (20-016-00124). 